La supraconductivité est

aujourd'hui une technologieclé des grands accélérateurs de particules, leur permettant d'atteindre des énergies de faisceau élevées tout en contenant leurs dimensions et leur consommation électrique. Les dispositifs supraconducteurs à basse température - aimants et cavités résonnantes hyperfréquence - requièrent des systèmes cryogéniques puissants à I'hélium liquide et, dans certains cas, superfluide. La cryogénie permet en outre d'assurer l'ultravide qui règne dans les tubes de faisceau et de garantir la stabilité des faisceaux.

Nous expliquons ces différents besoins, mentionnons les développements au cours du demi-siècle écoulé et présentons les défis des projets futurs.

Les mots ou expressions suivis d'un astérisque sont définis dans le glossaire, p. 26.

\title{
Cryogénie, supraconductivité et accélérateurs de particules : 50 ans de développements croisés
}

Philippe Lebrun (philippe.lebrun@cern.ch)

CERN, Organisation Européenne pour la Recherche Nucléaire, CH-1211 Genève 23, Suisse

$\mathrm{Au}$ cours des dernières décennies, la supraconductivité et la cryogénie associée sont devenues des technologies-clés des accélérateurs de particules de haute énergie, outils incontournables d'étude expérimentale de la physique (nucléaire, particules et matière condensée), qui comptent parmi les plus grands instruments construits par l'homme. Il suffit pour s'en convaincre d'observer l'évolution de la puissance totale de réfrigération cryogénique à la température de l'hélium liquide installée au CERN, l'Organisation Européenne pour la Recherche Nucléaire à Genève (fig. 1). Quelques grands réfrigérateurs cryogéniques d'hélium avaient fonctionné au CERN dès les années 1960, pour refroidir des chambres à bulles et des grands aimants d'analyse, mais ce n'est qu'avec l'apparition et le développement de la supraconductivité - cavités résonnantes hyperfréquence et aimants - dans les accélérateurs de particules que la puissance totale installée a crû vers les centaines de $\mathrm{kW}$ à $4,5 \mathrm{~K}$ que l'on connait aujourd'hui. Commençons donc par présenter les raisons qui ont fait pénétrer supraconductivité et cryogénie dans les accélérateurs de haute énergie.

\section{Le rôle de la supraconductivité et de la cryogénie dans les accélérateurs de particules}

Les accélérateurs sont des machines électromagnétiques, exerçant des forces sur des faisceaux de particules chargées par l'entremise de champs électriques et magnétiques pour les accélérer, les guider et les focaliser. L'absence de résistance électrique en courant continu, ou la dissipation ohmique limitée en courant variable dans les supraconducteurs permet d'augmenter le champ magnétique et ainsi de courber des faisceaux de plus haute

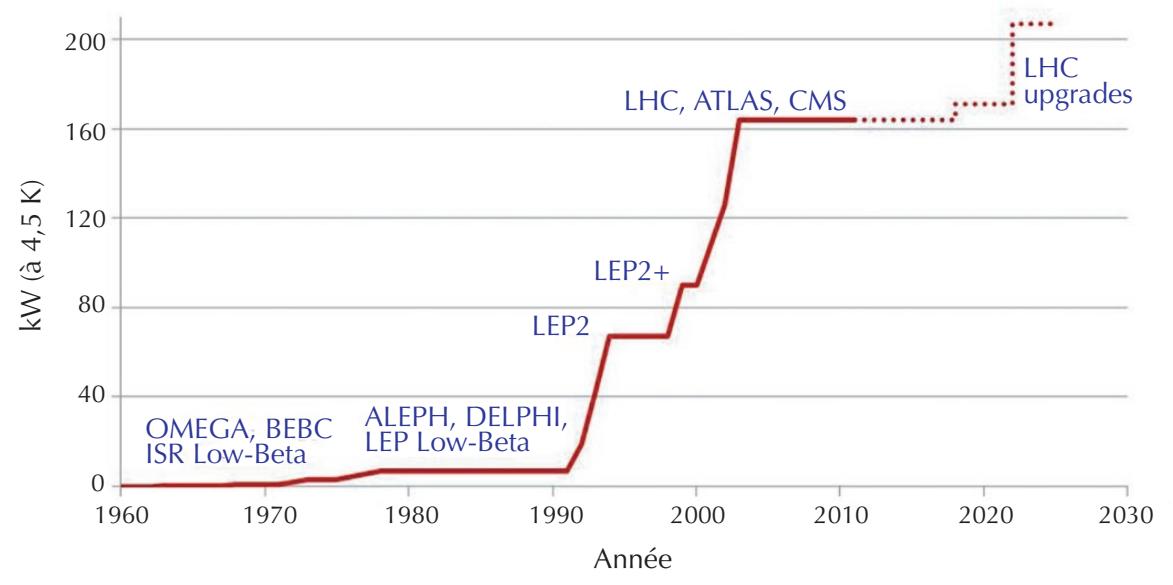

1. Puissance totale de réfrigération cryogénique à $4,5 \mathrm{~K}$ installée au CERN. OMEGA, BEBC, ALEPH, DELPHI, ATLAS et CMS sont de grands détecteurs de particules utilisant des spectromètres supraconducteurs, ISR Low-Beta et LEP Low-Beta sont des insertions à haute luminosité* équipant des collisionneurs, LEP2 et LHC sont des collisionneurs supraconducteurs. 


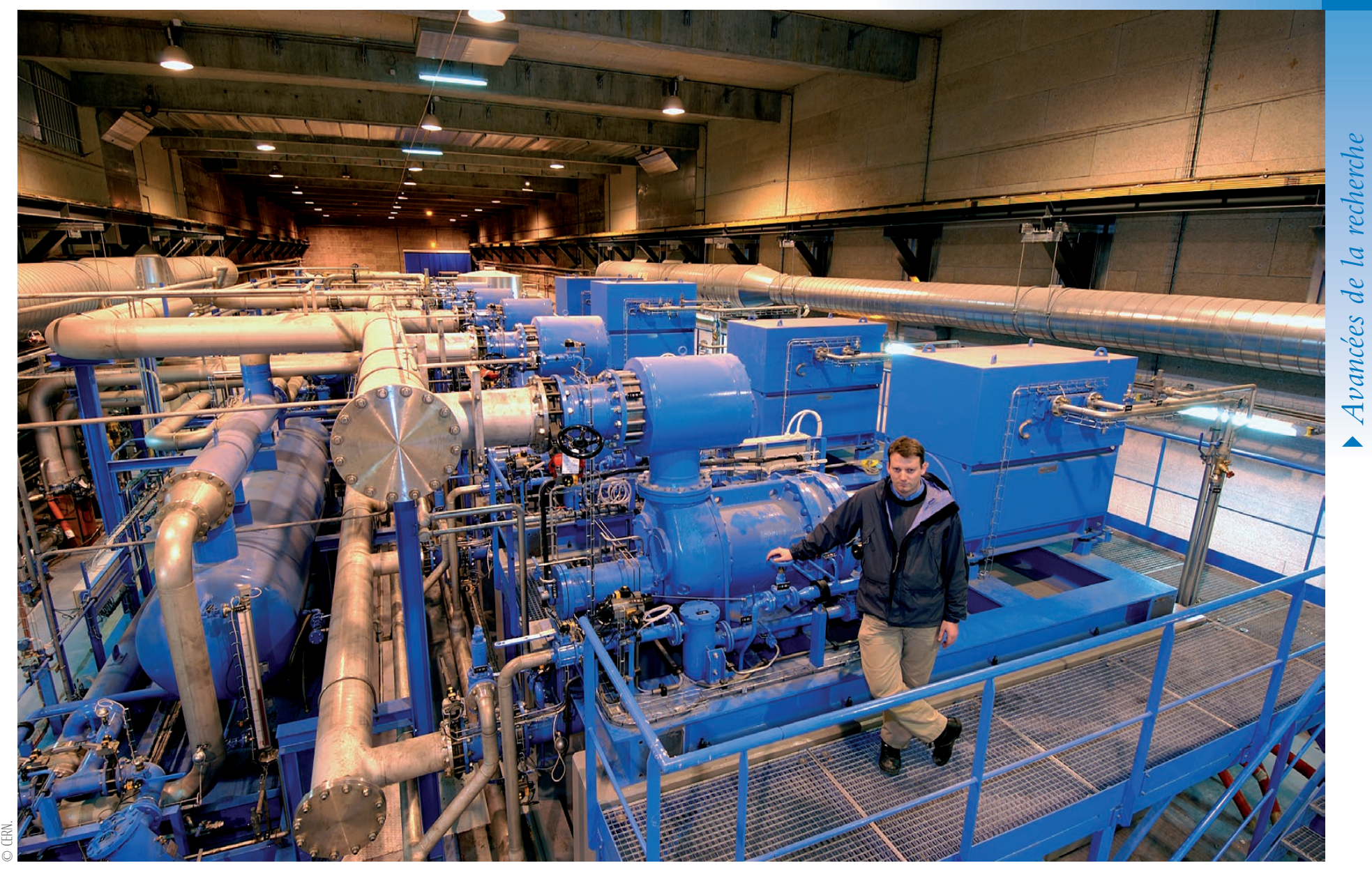

Compresseurs à vis lubrifiés du système cryogénique du LEP au CERN en 1996.

énergie pour un diamètre donné de l'accélérateur. Ce fut historiquement la première raison d'installer des aimants supraconducteurs dans les accélérateurs circulaires. Avec le développement de machines toujours plus grandes, la consommation électrique est devenue un enjeu majeur, justifiant par elle-même l'utilisation de dispositifs supraconducteurs pour économiser de l'énergie. C'est en particulier le cas des accélérateurs linéaires à onde continue* ou à fort facteur de charge $^{\star}$, dans lesquels la plupart de la puissance appliquée est dissipée dans la paroi des structures accélératrices hyperfréquence. C'est aussi le cas des systèmes magnétiques des très grands accélérateurs circulaires. Ces machines se sont développées selon deux axes pour pouvoir manipuler des faisceaux toujours plus rigides (fig. 2) : construire plus grand, et augmenter ainsi le rayon de courbure d'une part, et d'autre part augmenter le champ des aimants de guidage et de focalisation.

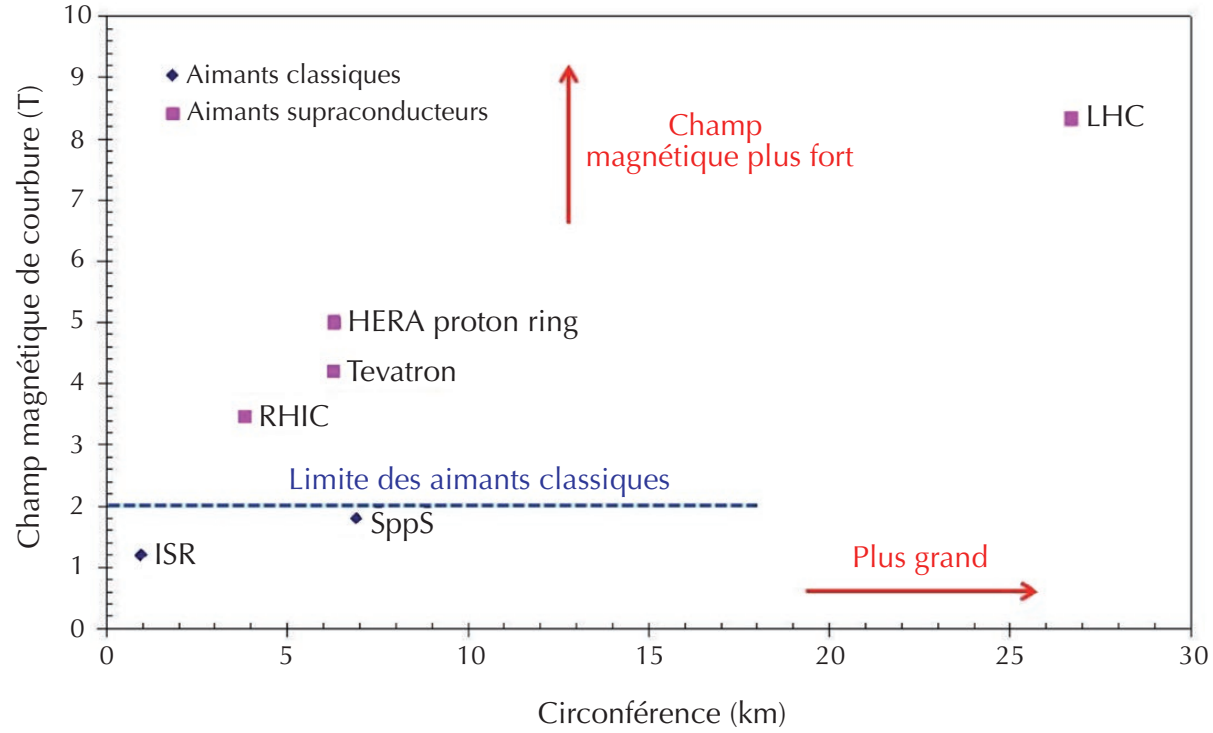

2. Axes de développement des grands accélérateurs circulaires de hadrons.

ISR : Anneaux de Stockage à Intersections (CERN). SppS : transformation du Super Synchrotron à Protons en collisionneur proton-antiproton (CERN). RHIC : Relativistic Heavy lon Collider (Brookhaven National Laboratory). Tevatron : collisionneur proton-antiproton (Fermilab). HERA proton ring : anneau de protons du collisionneur proton-électron HERA (DESY Hambourg). LHC : Grand Collisionneur de Hadrons (CERN). 


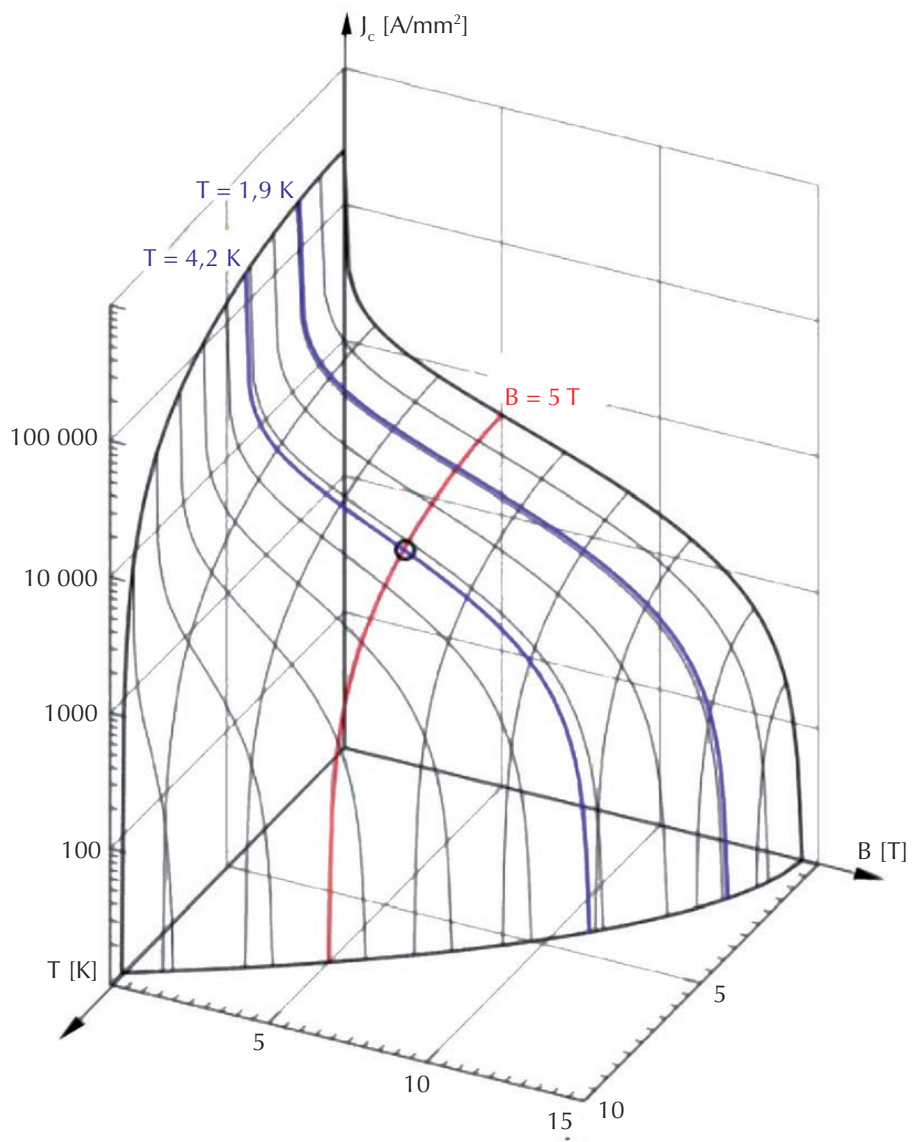

3. Surface critique du supraconducteur $\mathbf{N b}-\mathrm{Ti}$ : l'état supraconducteur règne sous la surface critique. L'abaissement de la température de fonctionnement de 4,2 K (hélium normal à saturation) à 1,9 K (hélium superfluide) élargit le domaine où règne l'état supraconducteur dans le plan champ magnétique B / densité de courant J.

\section{\〉}

La performance des aimants supraconducteurs est limitée par la surface critique* du matériau constitutif de leurs bobinages, surface qui sépare l'état supraconducteur de l'état normal dans l'espace température/ champ/densité de courant (fig. 3). Pour conserver suffisamment de capacité de transport de courant à champ élevé, le supraconducteur doit fonctionner à une fraction de sa température critique ${ }^{\star}$, en pratique à moins de la moitié. L'hélium liquide en ébullition normale à 4,2 K est donc le réfrigérant adapté à la plupart des aimants supraconducteurs d'aujourd'hui, utilisant des bobinages en alliage niobiumtitane caractérisé par une température critique $\mathrm{T}_{c}$ d'environ $9,5 \mathrm{~K}$.

La température critique d'un matériau diminue quand on augmente le champ magnétique. Pour aller au-delà de 10 teslas, on doit recourir à d'autres matériaux supraconducteurs que les alliages niobium-titane ( $\mathrm{Nb}-\mathrm{Ti})$, comme le composé intermétallique niobium-étain $\left(\mathrm{Nb}_{3} \mathrm{Sn}, \mathrm{T}_{\mathrm{c}}=18,3 \mathrm{~K}\right)$ ou les supraconducteurs "à haute température critique ${ }^{\star}$ ", que l'on ferait fonctionner dans l'hélium liquide : les difficultés de mise en œuvre (fragilité, sensibilité des performances à la déformation) de ces matériaux, ainsi que leur coût élevé par ampère transporté, en ont limité l'utilisation jusqu'ici.

Les aimants supraconducteurs d'un collisionneur de particules fonctionnant à champ constant ne présentent aucune dissipation d'énergie ; seule est à prendre en compte la puissance consommée par le système de réfrigération cryogénique, approximativement proportionnelle à la circonférence de la machine mais indépendante du champ produit par les aimants. En conséquence, la consommation spécifique d'électricité (kW par GeV d'énergie de faisceau) du système aimants supraconducteurs et cryogénie est inversement proportionnelle au champ magnétique (fig. 4). C'est donc à la fois la limitation du diamètre de la machine et de sa consommation d'électricité qui requièrent l'utilisation de la supraconductivité dans les collisionneurs circulaires de haute énergie.

L'argumentation est différente dans le cas des accélérateurs linéaires, essentiellement constitués de cavités accélératrices hyperfréquence (voir l'encadré, p. 23). Ces cavités sont des résonateurs pour lesquels on vise à maximiser le facteur de qualité` afin de réduire les pertes dans les parois, caractérisées par leur résistance superficielle. Alors que les cavités à paroi en cuivre à température ambiante présentent des facteurs de qualité de l'ordre de quelque $10^{4}$, les cavités supraconductrices peuvent atteindre des facteurs de qualité de $10^{9}$ à $10^{10}$ : la réduction de la puissance dissipée compense alors largement le coût thermodynamique de la réfrigération cryogénique. L'économie est d'autant plus importante que le facteur de charge ${ }^{\star}$ des cavités hyperfréquence est élevé, et maximale pour le fonctionnement en onde continue ${ }^{\star}$.

En outre, la résistance superficielle, qui contrôle la dissipation de puissance dans les cavités, a une composante proportionnelle au carré de la fréquence et à l'exponentielle du rapport de la température de fonctionnement à la température critique ${ }^{\star} d u$ supraconducteur utilisé. En conséquence, et en dépit du coût thermodynamique de la réfrigération à basse température, il peut se révéler avantageux de faire fonctionner des cavités supraconductrices hyperfréquence à des températures inférieures au point d'ébullition normal de l'hélium liquide, soit dans l'hélium superfluide` en-dessous de 2,2 K. Pour les cavités en niobium d'aujourd'hui, cela s'applique pour les fréquences supérieures à 700 ou $800 \mathrm{MHz}$. L'utilisation de niobium de plus grande pureté tend à déplacer cet optimum vers les plus basses températures. De manière converse, l'utilisation de supraconducteurs "à haute température critique * " réduirait sensiblement le terme exponentiel et permettrait de fonctionner dans l'hélium normal.

Outre le refroidissement de supraconducteurs, il peut se révéler nécessaire de faire fonctionner un accélérateur de particules à température cryogénique pour bénéficier de la faible résistivité électrique des conducteurs classiques, comme le cuivre. En effet, la circulation de particules chargées constitue un courant électrique qui induit des courants " images " dans la paroi métallique du tube de faisceau, produisant de la dissipation de puissance et des champs 


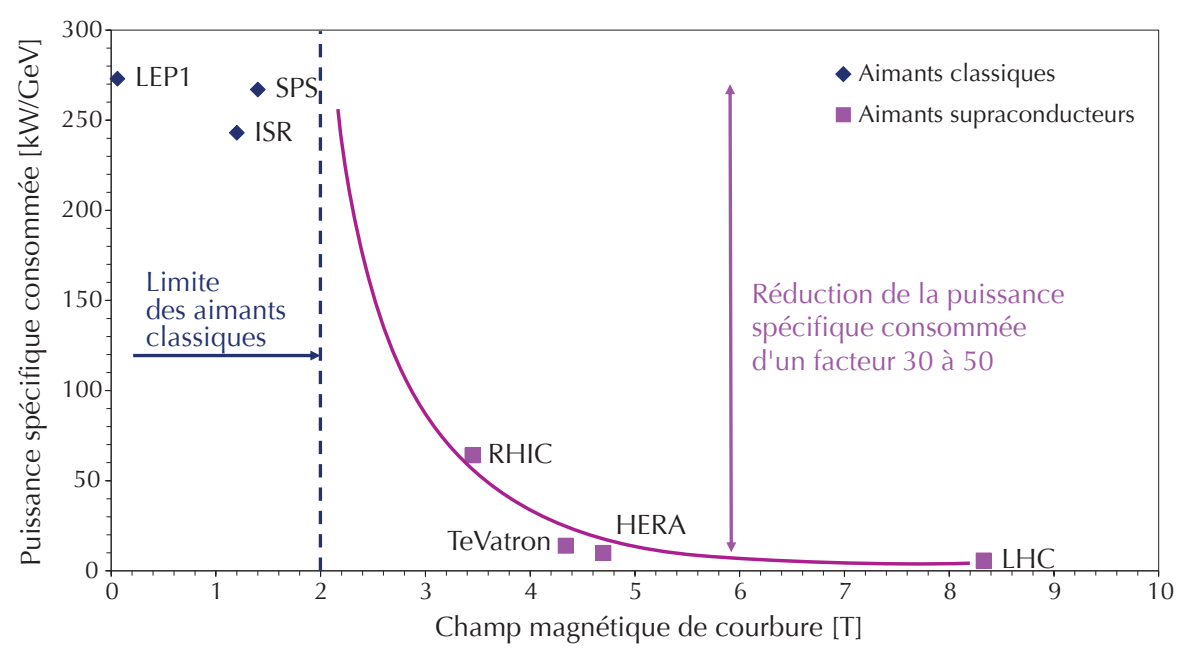

4. Puissance spécifique (kW/GeV d'énergie de faisceau) consommée par les aimants de courbure des collisionneurs circulaires. L'effet Joule dans les bobines d'aimants classiques, compte tenu de la densité de courant utilisée en pratique, est de l'ordre de $250 \mathrm{~kW} / \mathrm{GeV}$ d'énergie de faisceau. L'utilisation d'aimants supraconducteurs à champ élevé permet de réduire la circonférence des machines et proportionnellement la puissance cryogénique, quasi indépendante du champ de courbure.

\section{Cavités accélératrices hyperfréquence}

Dans les premiers accélérateurs de particules (Cockroft et Walton), le champ électrique accélérateur était fixe, et - pour des particules de charge unitaire - l'énergie de faisceau (en $\mathrm{eV}$ ) ne pouvait dépasser le champ électrique intégré (en $\mathrm{V}$ ) produit. En effet, le flux du champ électrique étant conservatif, l'accélération le long d'un parcours fermé est nulle et on n'aurait donc rien gagné à faire traverser plusieurs fois la structure accélératrice par le faisceau.

En 1924, G. Ising eut l'idée d'utiliser un champ électrique alternatif haute fréquence, synchrone avec le passage des particules dans la zone d'accélération, pour les accélérer de la valeur du champ intégré à chaque passage, et ainsi atteindre une énergie de faisceau bien au-delà de la tension accélératrice. La mise en œuvre pratique de cette idée se fait soit en multipliant les zones d'accélération en série l'une derrière l'autre (accélérateurs linéaires à " tubes de glissement » ou à chaîne de cavités résonnantes couplées), soit en faisant passer le faisceau un grand nombre de fois à travers une structure unique (la paire d'électrodes en D des cyclotrons) ou un petit nombre de structures (cavités résonnantes des synchrotrons). Pour limiter les pertes, il est en effet nécessaire de confiner le champ électromagnétique dans une cavité résonnante accordée à la fréquence souhaitée. Les structures accélératrices sont alimentées par des générateurs hyperfréquence, utilisant de l'électronique de puissance soit à tubes (tétrodes, klystrons) soit à l'état solide.

Le prix à payer pour l'accélération par champ variable est la dissipation de puissance dans les parois des cavités accélératrices, caractérisée par la résistance de surface du matériau utilisé ou, inversement par le facteur de qualité* du résonateur constitué par la cavité. Les cavités classiques sont en cuivre refroidi à l'eau, mais l'utilisation de supraconducteurs (refroidis à I'hélium) permet de réduire substantiellement la résistance de surface et, partant le bilan énergétique (y inclus le coût thermodynamique d'extraire la puissance dissipée à froid). Une autre manière de réduire la dissipation dans les cavités accélératrices est de n'y appliquer le champ que lorsqu'elles sont traversées par le faisceau ; en pratique, il faut aussi y ajouter le temps d'établissement du champ dans la structure. La fraction du temps pendant lequel la cavité est excitée s'appelle le facteur de charge*. Dans le cas de faisceaux intenses constitués de paquets de particules se succédant sans interruption, cette stratégie n'est évidemment pas applicable et on parle d'accélération à onde continue*. électromagnétiques qui interagissent avec les faisceaux en circulation. Cette interaction, caractérisée par une impédance proportionnelle à la résistivité électrique de la paroi, peut conduire à des instabilités de faisceau. Particulièrement importante pour les grands accélérateurs qui présentent des tubes de faisceau de petit diamètre, elle peut être compensée par des systèmes de contre-réaction rapide à condition que l'impédance reste limitée, d'où des contraintes sur le choix du matériau et la température de fonctionnement du tube de faisceau. Dans le Grand Collisionneur de Hadrons (LHC) du CERN, la première paroi "vue " par le faisceau est une couche de cuivre déposée sur le tube en acier austénitique, maintenu en dessous de $20 \mathrm{~K}$.

Un tube de faisceau à basse température constitue aussi une excellente cryopompe pour la plupart des gaz résiduels. La pression de saturation de tous les gaz, à part l'hélium, atteint des valeurs très faibles aux températures de l'hélium liquide, si bien que les accélérateurs supraconducteurs peuvent bénéficier de cet avantage. Pour éviter la désorption des molécules cryopompées par les rayonnements émis par le faisceau (rayonnement synchrotron et particules perdues), il peut s'avérer utile de protéger la paroi froide par un écran de faisceau. Dans le cas où la section efficace de diffusion des particules accélérées par les molécules des gaz résiduels est très grande (accélérateurs d'ions à fort niveau de charge électrique), la pression dans le tube de faisceau doit être maintenue extrêmement faible, ce qui devient l'argument principal pour que l'accélérateur soit cryogénique.

\section{Cinquante ans de projets et de progrès}

Bien que l'idée d'utiliser les supraconducteurs pour construire des aimants à champ élevé ait été formulée par H. Kamerlingh Onnes peu après la découverte de la supraconductivité en 1911, ce n'est qu'avec la disponibilité technique des supraconducteurs de seconde espèce ${ }^{\star}$ - qui tolèrent une pénétration partielle du champ magnétique - qu'elle put être mise en pratique : en 1960, J. Kunzler construisit et breveta un aimant supraconducteur de 1,5 $\mathrm{T}$ aux bobinages en alliage molybdène-rhénium, et c'est en 1961 que furent découverts les alliages niobium-titane qui équipent aujourd'hui la grande majorité des aimants supraconducteurs [1]. 
$>>$

Le potentiel de la supraconductivité et de la cryogénie pour augmenter les performances des accélérateurs linéaires, y compris l'option de refroidissement à l'hélium superfluide des cavités hyperfréquence [2], fut identifié dès les années 1960. Dans la même période, les avantages d'utiliser des aimants supraconducteurs dans les synchrotrons à protons furent clairement formulés et quantifiés en relation avec les projets de l'époque [3], et des développements techniques furent engagés dans le cadre du projet collaboratif européen GESSS, ainsi que dans des laboratoires russes et américains. Les technologies n'étaient pas matures et ces développements ne débouchèrent pas sur la construction d'accélérateurs supraconducteurs avant la décennie suivante.

Le premier système d'aimants supraconducteurs à être installé dans un accélérateur en exploitation, à la fin des années 1970, fut l'insertion à haute luminosité ${ }^{\star}$ des anneaux de stockage à intersections (ISR) du CERN, constituée de huit quadripôles focalisant les faisceaux et doublant ainsi la luminosité du collisionneur [4]. Le premier synchrotron entièrement supraconducteur fut le doubleur/économiseur d'énergie du Laboratoire National Fermi près de Chicago, qui entra en exploitation en 1983 et devint ensuite le collisionneur proton-antiproton Tevatron [5]. Le tunnel de 6,3 $\mathrm{km}$ de circonférence abritait 990 aimants supraconducteurs refroidis à $4,4 \mathrm{~K}$, construits dans le laboratoire. Ce projet pionnier inspira nombre de ses successeurs, dont le synchrotron à protons du collisionneur électron-proton HERA au laboratoire DESY à Hambourg, le premier accélérateur supraconducteur en Europe, et le premier au monde dont les aimants supraconducteurs furent entièrement construits par l'industrie [6]. La technologie se développa ensuite vers des machines toujours plus grandes, culminant avec le SuperCollisionneur Supraconducteur (SSC) aux USA [7] - projet de $83 \mathrm{~km}$ de circonférence qui fut abandonné en cours de construction - et le LHC au CERN, actuellement en exploitation, dont les 1800 aimants supraconducteurs principaux en $\mathrm{Nb}-\mathrm{Ti}$, répartis sur les $26,7 \mathrm{~km}$ du périmètre du tunnel, sont refroidis dans l'hélium superfluide à 1,9 K pour permettre leur fonctionnement jusqu'à 8,3 T, champ correspondant à l'énergie maximum de $7 \mathrm{TeV}$ des faisceaux de protons [8].

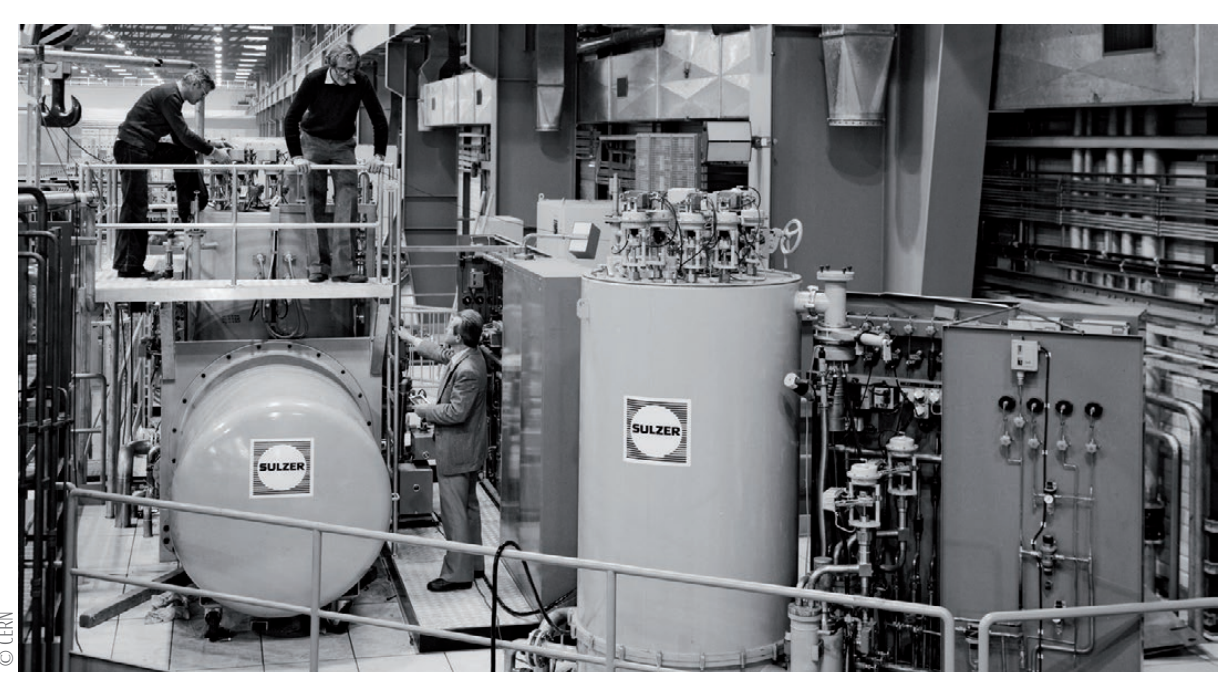

5. Réfrigérateurs $400 \mathrm{~W}$ à 4,5 K (Sulzer) alimentant les aimants des spectromètres de détecteurs de particules à la zone Nord du CERN dans les années 1970.
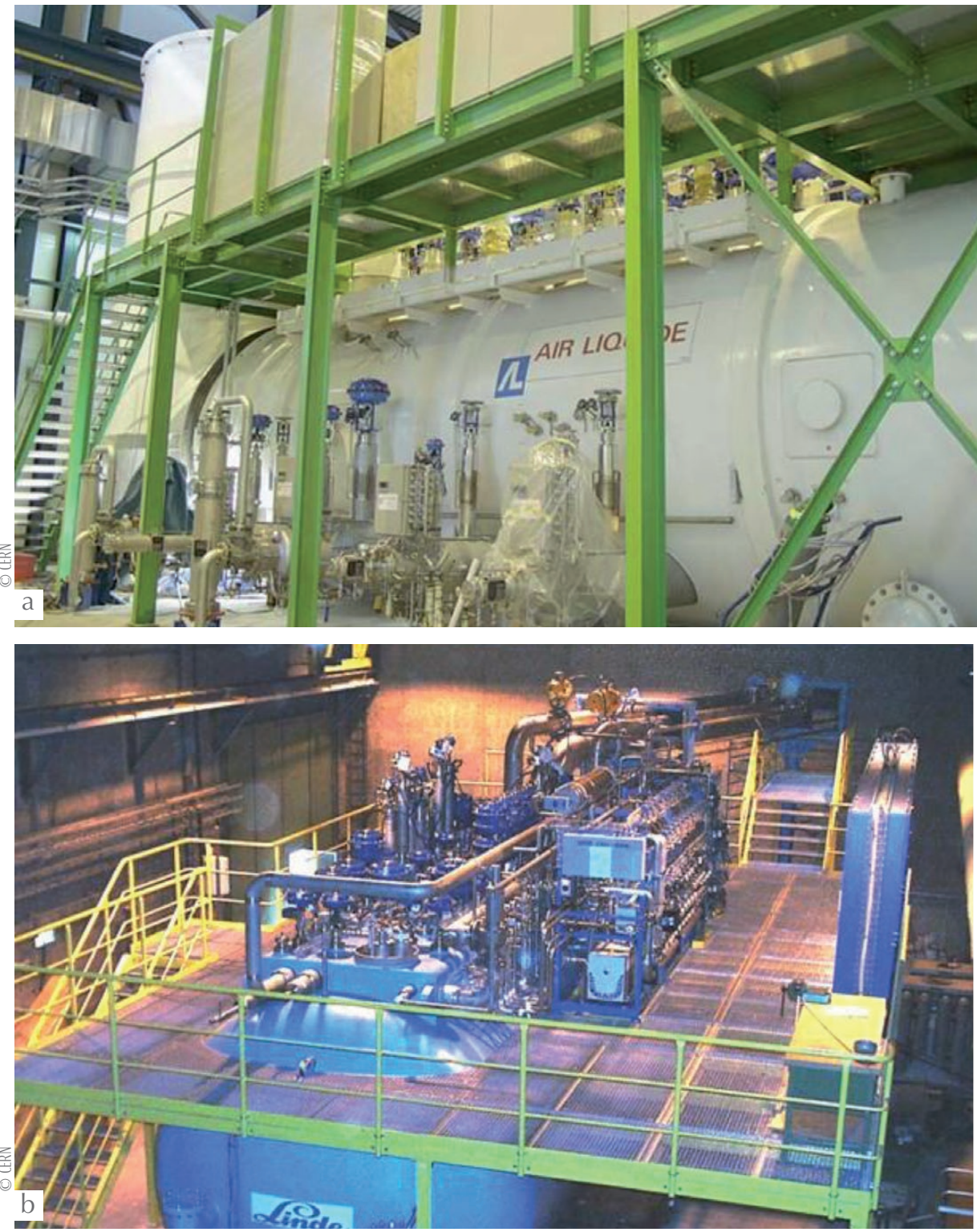

6. Réfrigérateurs 18 kW à 4,5 K (Air Liquide et Linde) alimentant (a) les cavités accélératrices hyperfréquence du LEP et (b) les aimants supraconducteurs du LHC dans les années 1990-2010. 
Des cavités hyperfréquence supraconductrices avaient été utilisées dès les années 1970 pour accélérer des électrons, mais c'est le projet CEBAF au Laboratoire National Thomas Jefferson à Newport News (USA) qui en fit la première application à grande échelle, dans ses deux linacs (accélérateurs linéaires) à recirculation utilisant des cavités en niobium à 1,5 $\mathrm{GHz}$ refroidies à $2 \mathrm{~K}$ [9]. Le plus grand système de cavités hyperfréquence supraconductrices réalisé à ce jour reste le système d'accélération du collisionneur électron-positon LEP, construit au CERN dans les années 1980, dont l'énergie de faisceau a graduellement été augmentée au cours des années 1990 (projet LEP2) par l'addition de 288 cavités supraconductrices $352 \mathrm{MHz}$ en niobium sur cuivre fonctionnant dans des bains d'hélium saturé à 4,5 K [10]. Ce projet nécessita l'installation autour de l'anneau de 26,7 km du LEP, de quatre grands réfrigérateurs d'une capacité initiale équivalente à $12 \mathrm{~kW}$ à $4,5 \mathrm{~K}$, portée ensuite à $18 \mathrm{~kW}$ à 4,5 K (fig. 6). Ces machines ont défini un nouveau standard de capacité et d'efficacité, et contribuent aujourd'hui à refroidir le LHC.

L'augmentation impressionnante des puissances unitaires des réfrigérateurs et liquéfacteurs d'hélium depuis les années 1970 - de quelques centaines de W à plusieurs dizaines de $\mathrm{kW}$ à $4,5 \mathrm{~K}$ (voir les figures 5 et 6 , et la courbe de la figure 1) - s'est accompagnée d'une amélioration substantielle de leur efficacité, qui atteint aujourd'hui 220 à $230 \mathrm{~W} / \mathrm{W}$ pour de grandes installations à capacité nominale, soit $28 \%$ du rendement de Carnot. Ce résultat important, compte tenu du fonctionnement quasi continu des installations cryogéniques et du prix croissant de l'énergie électrique, a été obtenu en agissant à la fois sur la configuration des cycles thermodynamiques et la performance individuelle des composants - compresseurs, échangeurs de chaleur, turbines de détente. Enfin, les progrès de l'instrumentation de procédé et la généralisation du contrôlecommande par ordinateur, utilisant des algorithmes complexes non linéaires contrôle adaptatif, contrôle prédictif basé sur des modèles physiques - mieux adaptés aux variations des propriétés de l'hélium et des matériaux à basse température, ainsi que la réalisation de simulateurs dynamiques pour la formation des opérateurs, ont permis une exploitation optimisée dans les différents modes de fonctionnement auxquels les installations de réfrigération cryogénique sont soumises.

Outre la production de froid, les projets d'accélérateurs de particules supraconducteurs ont aussi fait progresser tous les autres aspects de leur cryogénie : la conception des cryostats et la gestion des charges thermiques, les systèmes de distribution cryogénique, le stockage et la gestion de l'inventaire d'hélium, la frabilité opérationnelle et la maintenance préventive et curative.

Ce rapide survol d'un demi-siècle d'activité illustre le développement impressionnant de la supraconductivité et de la cryogénie dans les accélérateurs de particules : des techniques de laboratoire relativement exotiques se sont développées en des disciplines industrielles incontournables, au service d'un marché fixant des objectifs de performance, de fiabilité et de coût. Cette évolution est à mettre au crédit des instituts de recherche qui ont lancé des projets toujours plus ambitieux, mais aussi de l'industrie spécialisée qui a su répondre aux exigences du marché grâce à un progrès technique soutenu. Rappelons en particulier la position très compétitive de l'industrie française en cryogénie hélium.

\section{Un peu de prospective}

Un certain nombre d'accélérateurs supraconducteurs de haute énergie sont actuellement à l'étude ou en construction en Europe, en Asie et en Amérique. Utilisant des cavités accélératrices et des aimants supraconducteurs en grand nombre, ils ont des besoins importants de réfrigération cryogénique à la température d'ébullition normale de l'hélium ainsi que dans l'hélium superfluide. Les défis associés sont donc quantitatifs - champs électriques et magnétiques plus élevés, pertes électriques plus faibles en courant variable, plus grande capacité totale de réfrigération, inventaire hélium plus élevé - mais aussi qualitatifs - meilleure efficacité énergétique, meilleure fiabilité, plus grande adaptabilité à des charges variables, température de réfrigération plus basse dans le cas de cavités hyperfréquence utilisant de nouveaux matériaux supraconducteurs.

Parmi les accélérateurs linéaires supraconducteurs à haute intensité (à fort facteur de charge ${ }^{\star}$ ou à onde continue $\left.{ }^{\star}\right)$, il faut mentionner le linac à électrons de $17,5 \mathrm{GeV}$ alimentant le laser à électrons libres européen
Le dêveloppement impressionnant depuis un demi-siècle de la supraconductivité et de la cryogénie dans les accélérateurs de particules est à mettre au crédit des instituts de recherche, mais aussi de l'industrie qui a su répondre aux exigences du marché.g,

X-FEL de Hambourg, en cours de mise en service, le linac à électrons de $4 \mathrm{GeV}$ de la source de lumière LCLS II de Stanford, actuellement en projet, le linac à protons de $2 \mathrm{GeV}$ (puissance de faisceau $5 \mathrm{MW}$ ) de la source de neutrons de spallation européenne ESS en construction à Lund (Suède), le linac à ions $\mathrm{H}^{-}$de $800 \mathrm{MeV}$ du projet PIP II destiné à la production de faisceaux intenses de neutrinos au Fermilab, les linacs à ions $\mathrm{H}^{-}$et à ions lourds du Rare Isotope Science Project (RISP) à l'étude au laboratoire IBS de Deajeon (Corée du Sud). En préparation pour une construction possible au Japon, le collisionneur linéaire d'électrons et de positons de $500 \mathrm{GeV}$ ILC comporterait deux linacs se faisant face, équipés de 16000 cavités accélératrices, sur une longueur totale de $31 \mathrm{~km}$. Tous ces projets utilisent des cavités supraconductrices en niobium fonctionnant autour du $\mathrm{GHz}$, refroidies dans des bains d'hélium superfluide autour de $2 \mathrm{~K}$, pour produire économiquement des champs accélérateurs d'environ 20 à $30 \mathrm{MV} / \mathrm{m}$ avec des facteurs de qualité^ pouvant atteindre quelques $10^{10}$. Ils requièrent de puissantes installations cryogéniques produisant de quelques $\mathrm{kW}$ à quelques dizaines de $\mathrm{kW}$ à $2 \mathrm{~K}$.

Les accélérateurs circulaires de haute énergie demandent des aimants supraconducteurs toujours plus avancés, qu'il s'agisse de produire des champs magnétiques de courbure et de focalisation plus élevés, ou de limiter les pertes en champ variable. Ainsi, l'amélioration d'un ordre de grandeur de la luminosité du LHC (programme approuvé HL-LHC) requiert le remplacement des aimants de focalisation des 


\section{>>}

insertions à haute luminosité` actuels, en $\mathrm{NbTi}$, par des quadripoles à plus fort gradient et plus grande ouverture qui doivent être construits en $\mathrm{Nb}_{3} \mathrm{Sn}$. Bien que protégés par des collimateurs, ces aimants recevront des flux de particules beaucoup plus élevés résultant du halo de la collision des faisceaux, et de nouveaux réfrigérateurs cryogéniques dédiés seront nécessaires pour absorber la puissance thermique correspondante. À plus long terme, l'étude FCC (Future Circular Colliders), pilotée par le CERN et conduite en collaboration internationale, explore la faisabilité d'un très grand collisionneur de protons d'une énergie de collision de $100 \mathrm{TeV}$, qui aurait besoin d'aimants de courbure de $16 \mathrm{~T}$ combinant des bobines en $\mathrm{Nb}-\mathrm{Ti}$ et en $\mathrm{Nb}_{3} \mathrm{Sn}$, répartis sur un périmètre de $100 \mathrm{~km}$. Dans le cadre de cette étude, de nouveaux développements sont en cours en cryogénie de forte puissance, tels que le refroidissement de très longs secteurs (8 à $10 \mathrm{~km}$ ), l'utilisation de fluides caloporteurs non conventionnels, les cycles utilisant des mélanges héliumnéon avec récupération d'énergie sur les turbines de détente, l'impact de pressions de calcul plus élevées sur les performances thermiques des cryostats et lignes de distribution sous vide, et la conception d'installations de plus grande puissance de réfrigération, typiquement 50 à $100 \mathrm{~kW}$ à $4,5 \mathrm{~K}$ et jusqu'à $10 \mathrm{~kW}$ en-dessous de $2 \mathrm{~K}$. Les synchrotrons à cyclage rapide accélérant des protons et des ions - NICA en construction au JINR de Doubna (Russie), et SIS100 en construction au GSI de Darmstadt dans le cadre du projet FAIR - utilisent des aimants supraconducteurs à champ variable : leurs systèmes cryogéniques, essentiellement dimensionnés par les pertes dynamiques des aimants, ont aussi pour fonction de cryopomper les tubes de faisceau et d'atteindre le niveau d'ultravide requis pour limiter la diffusion des ions en circulation par les molécules de gaz résiduels.

Tous ces nouveaux projets constituent des opportunités uniques de développement technologique qu'il ne faut pas manquer pendant leur phase d'étude. Outre l'intérêt propre des projets, les progrès ainsi réalisés dans leurs technologies ancillaires comme la cryogénie et la supraconductivité appliquée profiteront à l'ensemble de ces disciplines.

Cet article est directement inspiré de la conférence du Prix Mendelssohn faite à Delhi en mars 2016.

\section{Glossaire}

Cryopompe : dispositif qui piège les molécules de gaz résiduel d'un système à vide par condensation ou sorption sur une surface froide.

Facteur de charge : fraction du temps pendant lequel le champ électromagnétique est présent dans une cavité accélératrice hyperfréquence.

Facteur de qualité d'un résonateur : $2 \pi$ fois le rapport entre l'énergie stockée dans un résonateur et l'énergie dissipée par période d'oscillation ; caractérise l'acuité de la résonance.

Hélium superfluide : phase liquide de l'hélium en-dessous de $2,2 \mathrm{~K}$; fluide quantique doté de propriétés de transport particulières.

Insertion à haute luminosité : ensemble de quadripôles réduisant les dimensions transverses des faisceaux d'un collisionneur au point de collision, pour augmenter la luminosité*.

Luminosité d'un collisionneur : nombre de réactions produites lors de la collision des faisceaux, par unité de temps et de section efficace.

Luminosité intégrée d'un collisionneur : nombre de réactions produites lors de la collision des faisceaux, par unité de section efficace.

Onde continue : mode de fonctionnement d'un accélérateur linéaire, où toutes les périodes du champ électrique servent à accélérer des paquets de particules.

Supraconducteur de seconde espèce : matériau tolérant la pénétration partielle du champ magnétique dans l'état supraconducteur, sous la forme d'un réseau de tubes de flux ; les supraconducteurs techniques utilisés dans les aimants sont de seconde espèce.

Surface critique d'un supraconducteur : dans l'espace température/champ magnétique/densité de courant électrique, surface en-dessous de laquelle règne l'état supraconducteur.

Température critique d'un supraconducteur : intersection de la surface critique* avec l'axe de la température ; température maximale à laquelle règne l'état supraconducteur, à champ magnétique et densité de courant nuls.

\section{Philippe Lebrun}

Philippe Lebrun travaille au CERN, I'Organisation Européenne pour la Recherche Nucléaire à Genève, sur les grands accélérateurs de particules. Il a dirigé le département « Technologies des Accélérateurs » du CERN pendant la construction du Grand Collisionneur de Hadrons LHC, projet utilisant massivement la cryogénie et la supraconductivité.

Membre de la division Accélérateurs de la SFP, il est aujourd'hui directeur de la Joint Universities Accelerator School (JUAS) et président de la Conférence Générale de I'Institut International du Froid.

Diplômé de l'École des Mines (Paris), du California Institute of Technology (Pasadena) et de l'Institut des Hautes Études pour la Science et la Technologie (Paris), Philippe Lebrun est docteur honoris causa de I'Université de Technologie de Wrocław (Pologne) et chevalier de la Légion d'honneur.

\section{Références}

1• J.K. Hulm et R.D. Blaugher, Phys. Rev. 123 (1961) 5.

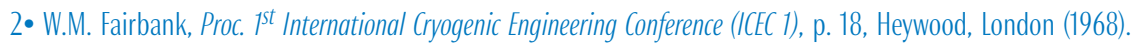

3• P.F. Smith et D. Lewin, Nucl. Inst. \& Meth. 52 (1967) 298.

4• J. Billan et al., IEEE Trans. Nucl. Sci. 26 (1979) 3179.

5• Tevatron phase 1, February, 1980, Rapport FERMILAB-DESIGN-1980-01 (1980).

6• HERA: a proposal for a large e-p colliding beam facility at DESY, Rapport DESY-HERA-81-10 (1981).

7• Conceptual design of the Superconducting Super Collider, Rapport SSC-SR-2020, SSC Design Group (1986)

8• LHC design report, Vol. 1: the LHC Main Ring, Rapport CERN-2004-003-V-1 (2004).

9• C.W. Leemann, The CEBAF superconducting accelerator: an overview, Rapport CEBAF-PR-86-003 (1986)

10• LEP design report, Vol. 3: LEP2, Rapport CERN-AC-96-01-LEP-2 (1996). 